\title{
FATORES ASSOCIADOS À OBESIDADE ENTRE TRABALHADORES DA SAÚDE NA BAHIA
}

\section{Eduardo Moreira Novaes Neto ${ }^{1}$ Tânia Maria de Araújo²; Camila de Carvalho Sousa $^{3}$}

1. Bolsista PIBIC/CNPq-Af, Graduando em Enfermagem, Universidade Estadual de Feira de Santana, email: eduardo.moreira18@ hotmail.com

2. Orientadora, Departamento de Saúde, Universidade Estadual de Feira de Santana, e-mail: araujo.tania@uefs.br

3. Doutoranda em Saúde Coletiva, Departamento de Saúde, Universidade Estadual de Feira de Santana, email: camilaxvii@ hotmail.com

PALAVRAS-CHAVE: Obesidade, Saúde do Trabalhador, Profissional de Saúde.

\section{INTRODUÇÃO}

A obesidade, fenômeno prevalente em grandes proporções em populações com padrões diversificados de cultura e desenvolvimento socioeconômico, constitui-se como importante problema de saúde pública (Boclin, 2010). Dados brasileiros mostram aumento considerável de obesidade na população adulta nas últimas décadas (Monteiro; Conde, 2000).

Uma das causas do aumento da obesidade na região das Américas, além das mudanças nos hábitos alimentares e redução da atividade física, são os avanços tecnológicos no trabalho, uso de veículos automotores e tempo gasto em atividades sedentárias como jogos, televisão e computadores (Peña, 2001).

Os trabalhadores apresentam frequentemente queixas de problemas de saúde relacionadas ao trabalho. Estas queixas estão ligadas tanto ao processo e condições de trabalho, como também aos aspectos característicos do ambiente, relações interpessoais e fatores relacionados ao próprio trabalhador, evidenciando assim, o modo pelo qual o trabalho pode influenciar nos aspectos biopsicossociais do trabalhador (Bohle et al., 2004).

As doenças que acometem o sistema endócrino, entre elas a obesidade, podem afetar a saúde dos trabalhadores e, então, a atividade profissional exercida. Do mesmo modo que a atividade laboral pode alterar o sistema endócrino, contribuindo para o surgimento da obesidade. Em contrapartida, o estilo de vida do trabalhador poderá amenizar ou aumentar a gravidade desse problema (Saad et al., 2006).

Dentre as consequências que o excesso de peso pode trazer, destacam-se a diminuição da qualidade de vida, aumento do uso de cuidados com a saúde, diminuição da produtividade no ambiente de trabalho e aumento do absenteísmo (Hofelmann, 2009; Magalhais; Yassaka; Soler, 2008).

Descrever a obesidade, bem como identificar os grupos de risco específicos são ferramentas importantes para a promoção da saúde, diante da heterogeneidade dos espaços sociais. Por isso, as possíveis relações entre obesidade e processos de trabalho diversos são consideradas importantes temáticas de estudo, porém, pouco explorado entre os trabalhadores do Brasil (Boclin, 2010), sobretudo entre os trabalhadores da saúde.

Este estudo justifica-se pela necessidade de dimensionar adequadamente a prevalência da obesidade entre trabalhadores da saúde no contexto da Atenção Básica e da Média complexidade a fim de evidenciar o perfil epidemiológico deste desfecho para subsidiar estratégias de combate, bem como promover a saúde dos trabalhadores da saúde. 
O objetivo deste estudo foi estimar a prevalência da obesidade entre trabalhadores da saúde, descrever as características sociodemográficas, hábitos de vida, características do trabalho e aspectos psicossociais do trabalho e identificar os principais fatores associados a obesidade entre os trabalhadores da saúde na Bahia.

\section{MATERIAL E MÉTODOS}

Estudo epidemiológico de corte transversal, exploratório. Constitui um recorte da pesquisa "Condições de Trabalho, Condições de Emprego e Saúde dos Trabalhadores da Saúde na Bahia", realizada pelo Núcleo de Epidemiologia da Universidade Estadual de Feira de Santana, no ano de 2012.

A população do estudo foi composta por 6.191 trabalhadores da saúde dos municípios de Feira de Santana, Santo Antônio de Jesus e Itabuna.

O cálculo do tamanho amostral, com a finalidade de verificar o poder do estudo para análise pretendida, uma vez que os dados já haviam sido coletados, foi realizado empregando a fórmula para a população finita. Para a estimativa, considerou-se o total de trabalhadores $(\mathrm{N}=6.191)$, a prevalência de $17,7 \%$ de obesidade entre trabalhadores da saúde (Sousa et al., 2007), precisão de $\pm 4 \%$, nível de confiança de $95 \%$, resultando em 322 trabalhadores. Acresceu-se 20\% $(n=65)$ para perdas e recusas, sendo a amostra final calculada em 387. O cálculo foi realizado utilizando o software Epi Info, versão 6.04d. A fim de aumentar o poder do estudo, foram utilizados todos os 3.014 trabalhadores para os quais os dados foram coletados no estudo multicêntrico.

Para a coleta de dados foi utilizado um questionário construído com base na revisão da literatura e testado em estudo piloto. $O$ instrumento foi composto por questões sobre características sociodemográficas, do emprego, condições de trabalho e aspectos psicossociais do trabalho, atividades domésticas e hábitos de vida.

A variável de desfecho foi definida segundo morbidade autorreferida, obtida pela questão: possui diagnóstico médico de Obesidade? Assim, neste estudo, foi considerado caso de Obesidade a resposta afirmativa (sim) à essa questão.

Como variáveis de exposição foram analisadas: a) Características sociodemográficas; b) Características gerais do trabalho; c) Hábitos de vida; d) Aspectos psicossociais do trabalho (estressores ocupacionais) - estes aspectos foram avaliados utilizando-se o JCQ - Job Content Questionnaire, previamente testado (Araújo; Karasek, 2008).

Foram realizadas análises univariada, bivariada e multivariada. $\mathrm{Na}$ análise univariada foram apresentadas as frequências brutas e relativas para cada uma das variáveis selecionadas para análise. $\mathrm{Na}$ análise bivariada foram estimadas as razões de prevalência com seus respectivos intervalos de confiança a 95\% para a associação entre as variáveis de exposição e a Obesidade.

$\mathrm{Na}$ pré-seleção das variáveis para a análise multivariada foi empregado o teste do Qui-Quadrado de Person $\left(\mathrm{X}^{2}\right)$ ou exato de Fisher para avaliar associações entre exposição e desfecho de interesse. O nível de significância para entrada no modelo multivariado foi de $p \leq 0,25$. Para a análise multivariada foi utilizada a regressão logística não condicional. As associações foram consideradas significantes ao nível de $5 \%(\mathrm{p} \leq 0,05)$.

Os dados foram analisados no software "Statistical Package for the Social Science" (SPSS), versão 23.0 e "Data Analysis and Statistical Software" (STATA), versão 12.0 .

Os protocolos de pesquisa foram avaliados e aprovados pelo Comitê de Ética em Pesquisa com Seres Humanos da Universidade Estadual de Feira de Santana sob parecer no081/2009. Os profissionais selecionados para o estudo após concordarem em participar, assinaram o Termo de Consentimento Livre e Esclarecido (TCLE). 


\section{RESULTADOS E DISCUSSÃO}

Dentre os entrevistados, $78,0 \%$ eram mulheres. Grande parte $(58,1 \%)$ dos profissionais possuía idade superior a 35 anos. A maioria $(79,7 \%)$ declarou-se preto ou pardo, quando questionados sobre cor da pele autorreferida. O nível de escolaridade de maior percentual foi o nível superior $(43,0 \%)$, seguido do nível fundamental e médio $(40,2 \%)$. Sobre a situação conjugal, $57,3 \%$ possuíam companheiro(a). A maioria $(68,6 \%)$ tinha filhos, dentre estes, 73,0\% possuíam entre um e dois filhos. Quando indagados sobre sua renda mensal, $70,3 \%$ relataram receber até 1.000 reais mensalmente.

De acordo com as características ocupacionais, 58,5\% dos trabalhadores possuíam vínculo empregatício do tipo efetivo. A maioria $(65,2 \%)$ tinha até 10 anos de tempo de trabalho. No que diz respeito a jornada de trabalho, elevado percentual $(77,2 \%)$ relatou trabalhar 40 horas semanais ou menos. O turno de trabalho mais frequente foi o diurno $(79,8 \%)$, que correspondia aos trabalhos no turno da manhã e tarde.

Sobre os hábitos de vida dos trabalhadores, 56,1\% relataram não praticar atividade física. Quando perguntados sobre atividades de lazer, 83,6\% relataram ter tais atividades. Dentre as atividades de lazer encontravam-se: assistir TV, ouvir rádio, atividades culturais e sociais. No que diz respeito ao tabagismo, 6,0\% tinham o hábito de fumar. Sobre o consumo de bebidas alcoólicas, 39,1\% possuíam este hábito.

No que diz respeito ao estresse ocupacional, 21,8\% dos trabalhadores foram classificados no grupo de alta exigência, sendo este estrato o de maior risco à saúde.

A prevalência de Obesidade entre os trabalhadores da saúde da Atenção Básica e Média Complexidade foi de 10,7\%. Após regressão logística não condicional, permaneceram associadas a Obesidade: sexo, ter filhos, ter mais de dois filhos, ausência de prática de atividade física e consumo de bebida alcoólica.

A prevalência da Obesidade entre trabalhadores da saúde apresenta resultados similares aos encontrados em outros estudos. Em pesquisa realizada com funcionários plantonistas de unidades de saúde de Teresina, no Piauí, evidenciou-se prevalência de obesidade de 17,7\% (Sousa et al., 2007). Porém, num estudo feito com trabalhadores de enfermagem de unidades de cuidados intensivos e emergência em um hospital de pronto-socorro da região sul, foi encontrada prevalência de obesidade $31,1 \%$ (Silveira et al., 2013).

A associação entre obesidade e sexo feminino pode ser explicada biologicamente visto que as mulheres possuem maior acúmulo de gordura visceral (Who, 1995; Silveira; Lopes; Caialfa, 2007). Porém, deve-se levar em consideração que a mulher, diante de sua ascensão social na contemporaneidade, bem como aumento da carga horária total de trabalho (trabalho profissional e trabalho doméstico), pode apresentar-se mais exposta a hábitos de vida não saudáveis como a ausência da prática de atividade física e alimentação não saudável, expondo mais as mulheres a situações que podem contribuir para a obesidade, bem como para maior risco cardiovascular.

O fato do trabalhador da saúde ter filhos, bem como ter mais de dois filhos permaneceu associado a obesidade pode ser explicado diante da responsabilidade que tais trabalhadores são demandados no cuidado aos filhos, bem como ao aumento das despesas financeiras, conduzindo-os a trabalharem cada vez mais a fim de atender esta demanda. $\mathrm{O}$ aumento da carga horária de trabalho, bem como a aquisição de mais de um vínculo empregatício podem contribuir para o surgimento de hábitos não saudáveis e estresse ocupacional, contribuindo, dentre outras consequências, para a ocorrência de obesidade. 


\section{CONCLUSÃO}

A prevalência da obesidade entre os trabalhadores da saúde foi de $10,7 \%$, sendo este desfecho associado a pertencer ao sexo feminino, ter filhos, ter mais de dois filhos, não praticar atividade física e consumir bebida alcoólica.

A obesidade constitui-se um problema sério que precisa de intervenções entre trabalhadores da saúde, diante das consequências que pode trazer tanto para o trabalho quanto para a saúde do trabalhador. Ações simples como mudanças no trabalho e nos hábitos de vida contribuem para a prevenção e tratamento deste desfecho. Essas evidências podem instrumentalizar equipes de educação permanente e gestores para a adoção de estratégias de enfrentamento.

Este estudo apresenta algumas limitações. Dentre elas destaca-se o efeito do trabalhador sadio, no qual somente os trabalhadores mais resistentes permanecem no trabalho, contribuindo para a subestimação do desfecho.

\section{REFERÊNCIAS}

ARAÚJO, T.M.; KARASEK, R. Validity and reliability of the job content questionnaire in formal and informal jobs in Brazil. Scandinavian Journal of Work Environment \& Health, Helsinki, v. 34, supl. 6, p. 52-59, 2008.

BOCLIN, K. L. S; BLANK, N. Prevalência de sobrepeso e obesidade em trabalhadores de cozinhas dos hospitais públicos estaduais da Grande Florianópolis, Santa Catarina.

Rev. bras. Saúde ocup, São Paulo, v. 35, n. 121, p. 124-130, 2010.

MONTEIRO, C. A.; CONDE, W. L.; CASTRO, I. R. R. A tendência cambiante da relação entre escolaridade e risco de obesidade no Brasil (1975-1997). Cad Saúde Pública, Rio de Janeiro, v. 19, Sup. 1, p. S67-S75, 2003.

PEÑA, M; BACALLAO, J. La obesidad y sus tendências en la región. Rev Panam Salud Pública, v. 10, n. 2, p. 75-78, 2001.

BOHLE, P; QUINLAN, M; KENNEDY, D; WILLIAMSON, A. Working hours, worklife conflict and health in precarious and "permanent" employment. Rev Saúde Pública, v. 19, p. 19-25, 2004.

SAAD, M. J. A; ZANELLA, M. T; FERREIRA, S. R. Síndrome metabólica: ainda indefinida, mas útil na identificação do alto risco cardiovascular. Arq Bras Endocrinol Metab, v. 50, n. 2, p. 161-162, 2006.

HOFELMANN, D. A; BLANK, N. Excesso de peso entre trabalhadores de uma indústria: prevalência e fatores associados. Rev Bras Epidemiol, v. 12, n. 4, p. 657670, 2009.

MAGAlHAIS, L. C. B; YASSAKA, M. C. B; SOLER, Z. A. S. G. Indicadores de qualidade de vida no trabalho entre docentes de curso de graduação em enfermagem. Arq Ciênc Saúde, v. 15, n. 3, p. 117-124, 2008.

SOUSA, R. M. R. P. et al. Prevalência de sobrepeso e obesidade entre funcionários plantonistas de unidades de saúde de Teresina, Piauí. Rev. Nutr, v. 20, n. 5, p. 473-482, 2007.

SILVEIRA, C. D. S. et al. Perfil de sobrepeso e obesidade em trabalhadores de enfermagem em unidades de cuidado intensivo e emergência. Revista Ciência $\&$

Saúde, v. 6, n. 3, p. 157-162, 2013.

WORLD HEALTH ORGANIZATION. Physical status: the use and interpretation of anthropometry. Geneva: World Health Organization, 1995.

SILVEIRA, E. A; LOPES, A. C. S; CAIALFA, W. T. Avaliação do estado nutricional de idosos. In: KAC, G; SICHIERI, R; GIGANTE, DP (Org.). Epidemiologia

nutricional. Rio de Janeiro: Editora Atheneu; 2007. p. 105-25. 